Original Paper http://ajol.info/index.php/ijbcs http://indexmedicus.afro.who.int

\title{
Caractéristiques de la végétation du parcours Gadoudhé, dans la commune rurale de Fabidji (Niger)
}

\author{
Ibrahim DJIBO ${ }^{1,5^{*}}$, Mani MAMMAN ${ }^{2}$, Chaibou ISSA ${ }^{3}$, Oumar SARR ${ }^{4}$, \\ Amy BAKHOUM ${ }^{4}$, Hamani MARICHATOU ${ }^{1}$, E. Léonard $\mathrm{AKPO}^{4}$ et Moussa ASSANE \\ ${ }^{1}$ Université Abdou Moumouni, Faculté d'Agronomie, laboratoire d'insémination artificielle et de production \\ d'azote liquide, $B P n^{\circ} 10960$ Niamey, Niger. \\ ${ }^{2}$ Institut National de la Recherche Agronomique du Niger, Département productions animales, BP $n^{\circ} 429$ \\ Niamey, Niger. \\ ${ }^{3}$ Université Dan Dicko Dankoulodo de Maradi, BP $n^{\circ} 465$ Maradi, Niger. \\ ${ }^{4}$ Université Cheick Anta Diop, Faculté des sciences et Techniques, laboratoire d'Écologie et d'Ecohydrologie, \\ $B P n^{\circ} 5005$ Dakar - Fann, Sénégal. \\ ${ }^{5}$ Université Cheick Anta Diop, École Inter-États des Sciences et Médecines Vétérinaires, laboratoire de \\ physiologie et de pharmacodynamie, BP $n^{\circ} 5077$ Dakar - Fann, Sénégal. \\ *Auteur correspondant ; E-mail: maiga2222@yahoo.com
}

\section{RESUME}

Cette étude sur les caractéristiques de la végétation (herbacée et ligneuse) du parcours Gadoudhé a été conduite dans la commune rurale de Fabidji (Niger). Elle a été menée à l'intérieur de 126 placettes (de 2500 $\mathrm{m}^{2}$ ) posées le long de 4 transects (de $3,3 \mathrm{~km} ; 2,7 \mathrm{~km} ; 2,3 \mathrm{~km}$ et 4,3 km). La méthode des points quadrats alignés a permis de recenser les herbacés. La phytomasse herbacée a été évaluée par la méthode de la récolte intégrale. Après avoir établi la liste des espèces ligneuses, des mesures sur les paramètres dendrométriques ont été réalisées. La régénération des espèces ligneuses a été appréciée par le comptage des rejets vivants et morts. Un total de 37 espèces végétales ont été inventoriées dont 27 sont des herbacés. Les familles des herbacés les plus représentées sont les Poacées, les Fabacées, les Amaranthacées et les Convolvulacées. La phytomasse résiduelle est de 471,1 kg MS/ha, avec une valeur pastorale nette de 60,20\% qui correspond à 283,61 $\mathrm{kg}$ de MS/ha de fourrage « qualifié », soit une capacité de charge estimée à 0,041 UBT/ha/an. Quant aux ligneux, ils sont représentés par 10 espéces dont principalement des combrétacées. La structure du peuplement montre des individus de hauteur et de diamètre moyens. La surface terrière a été évaluée à $0,01 \mathrm{~m}^{2} / \mathrm{ha}$ pour une densité de 13,75 individus à l'hectare. La régénération des jeunes plants présente une densité de 1020,91 rejets à l'hectare. Les résultats obtenus, mettent en évidence la nécessité de penser à un aménagement du parcours afin d'assurer sa durabilité.

(C) 2018 International Formulae Group. All rights reserved.

Mots clés: Herbacés, Ligneux, Capacité de charge, Paramètres dendrométriques, Parcours Gadoudhé, Niger. 


\title{
Characteristics of the Gadoudhé course vegetation in the rural area of Fabidji (Niger)
}

\begin{abstract}
This study on the characteristics of the vegetation (herbaceous and woody) Gadoudhe course was conducted in the rural area of Fabidji (Niger). Within 126 plots that have been set through 4 transects. Aligned quadrat points method allowed taking an inventory of the herbaceous. The herbaceous biomass was evaluated by the method of the full harvest. After making the list of woody species, the measures on dendrometric parameters were conducted. The regeneration of the species has been appreciated by the count of live and dead discards. 37 were inventoried. Among these 27 species are herbaceous plants. The most represented families of grasses are the Poaceae, Fabaceae, Amaranthaceae and the Convolvulaceae. Residual biomass is $471.1 \mathrm{~kg}$ MS/ha of « qualified » fodder. What gives an estimated carrying capacity to $0.041 \mathrm{UBT} / \mathrm{ha} / \mathrm{year}$. As for the wood, they are represented by 10 species, especially represented by the Combretaceae. The structure of the settlement shows average individuals of height and diameter. Basal area was evaluated to $0.01 \mathrm{~m}^{2} / \mathrm{ha}$ at a density of 13.75 individuals per hectare. The regeneration of seeding presents a density of releases per hectare 1020.91. The results obtained highlight the need to think about a layout of the route to ensure its sustainability. (C) 2018 International Formulae Group. All rights reserved.
\end{abstract}

Keywords: Herbaceous- Woody- Carrying capacity- Dendrometric parameters-Gadoudhé course-Niger.

\section{INTRODUCTION}

Les fourrages tropicaux présentent un intérêt grandissant pour l'alimentation animale, vue la progression de la demande en produits animaux qui devrait doubler dans les Pays du Sud, contrairement au Nord où la demande devrait en revanche stagner (FAO, 2003 ; FAO 2006).

Au Sahel, c'est l'intensité de la saison des pluies et sa répartition spatiale qui déterminent le stock potentiel de fourrage disponible au cours de la longue saison sèche qui suit (Garba et al., 2009). Or on assiste, avec la récurrence des déficits pluviométriques à une réduction en quantité et en qualité du disponible fourrager des pâturages pour l'alimentation du bétail (Uwizéyé, 2008).

$\mathrm{Au}$ Niger, même si les espaces pastoraux favorables à l'élevage couvrent une superficie de $620000 \mathrm{~km}^{2}$ (Maidadji, 2003; Rhissa, 2010 ; Chaibou et al., 2012), la faible productivité fourragère des parcours liée à l'irrégularité des pluies, constitue une contrainte majeure à l'élevage. En effet, le bilan fourrager établi par les services d'élevage sur les dix (10) dernières années a fait ressortir cinq (5) années déficitaires (Rhissa, 2010). Par ailleurs, le cheptel du Niger, est confronté à un problème alimentaire lié à l'amenuisement des espaces pastoraux au profit des cultures.

C'est dans ce contexte que, la commune rurale de Fabidji (Région de Dosso au Niger) a élaboré un schéma d'aménagement foncier dans le but de sécuriser le parcours Gadoudhé comme principale localité de pâture pour le bétail.

Cette étude vise principalement à établir les caractéristiques de la végétation dudit parcours. De manière spécifique, il s'agit de faire un inventaire floristique de la végétation (herbacée et ligneuse) et de décrire certains paramètres (capacité de charge, valeur pastorale, surface terrière, couvert aérien, densité) de cette végétation.

\section{MATERIEL ET METHODES}

\section{Présentation du site de l'étude}

L'étude a été menée dans la commune rurale de Fabidji, dans le parcours Gadoudhé (Figure 1) d'une superficie de 3590 ha. Cette commune rurale est l'une des 8 communes du département de Boboye et compte 29 villages ; elle est située au Sud-Ouest du cheflieu du département (Région de Dosso) entre $12^{\circ} 25^{\prime}$ et $13^{\circ} 10^{\prime}$ Latitude Nord et $2^{\circ} 30^{\prime}$ et $2^{\circ} 57^{\prime}$ Longitude Est et couvre une superficie de $1075 \mathrm{Km}^{2}$ (PDC, 2007). L'effectif de la population est de 45405 habitants dont la 
moitié $(50,3 \%)$ est composée de femmes. La densité moyenne est de 43,86 habitants/ $\mathrm{Km}^{2}$ (INS, 2014). L'élevage est la seconde activité pratiquée après l'agriculture et permet de combler le déficit céréalier de la campagne agricole. En 2017, le cheptel de la zone (département de Boboye) est estimé à 530390 têtes, toutes espèces confondues soit 226039 UBT (RGAC, 2007).

Le climat de la zone d'étude est caractérisé par une longue saison sèche de 8 mois (allant des mois d'octobre à mai) et une courte saison pluvieuse de 4 mois (juin à septembre). La pluviométrie est très variable (550 $\mathrm{mm}$ au Nord à $800 \mathrm{~mm}$ au Sud) et mal repartie dans l'espace et le temps. Les relevés pluviométriques des 8 dernières années (20092016) confirment cette variabilité (Figure 2) avec une moyenne de $550,91 \mathrm{~mm}$ de pluies tombées en 26,87 jours.

\section{Echantillonnage}

Compte tenu de la forme irrégulière de l'aire de pâturage, 4 transects de longueurs variables $(3,3 \mathrm{~km} ; 2,7 \mathrm{~km} ; 2,3 \mathrm{~km}$ et 4,3 $\mathrm{km}$ ), orientés dans la direction Nord-Sud et espacés de 1,5 km ont été tracés. Le long de ces transects, des placettes de $2500 \mathrm{~m}^{2}$ correspondant à l'aire minimale en zone sahélienne (Boudet, 1984) ont été posées par intervalle de $50 \mathrm{~m}$. Au total 126 placettes ont été disposées sur les 4 transects. C'est à l'intérieur de ces placettes que l'ensemble des données ont été collectées.

\section{Collecte des données}

\section{Recensement des espèces herbacées}

Pour identifier les espèces herbacées de l'aire de pâturage, la méthode des points quadrats alignés proposée par Daget et Poissonnet (1971) a été utilisée. La méthode a consisté à recenser la présence des espèces herbacées à la verticale de 100 《points » positionnés sur une ficelle de $20 \mathrm{~m}$ de longueur graduée tous les $20 \mathrm{~cm}$ et tendue audessus du tapis herbacé. On déplace perpendiculairement au sol, le long de cette ficelle, une tige métallique fine(ou ligne de visée) qui est à chaque fois descendue jusqu'au sol. On enregistre toutes les espèces herbacées qui sont en contact avec la ligne de visée. Plusieurs espèces peuvent être notées au même point de lecture mais par convention chaque espèce n'est recensée qu'une seule fois. Si en revanche aucune espèce végétale ne touche la tige métallique on note la présence d'un sol nu. Pour chaque placette, 4 lignes de 100 points chacune (soient 400 points de lecture) ont été effectuées le long d'une médiane. Les données recueillies ont permis de calculer les fréquences et contributions spécifiques des espèces herbacées recensées.

\section{Estimation de la phytomasse herbacée}

La phytomasse herbacée a été évaluée par la méthode de la récolte intégrale (Devineau et al ; 1984 ; Daget et Godron, 1995). Elle consiste à poser des carrés de $1 \mathrm{~m}$ de côté et à couper au ras de sol toute la phytomasse aérienne herbacée en prenant la précaution de ne pas déraciner l'herbe. Les carrés sont disposés sur les 4 angles et au milieu de la placette. Ce qui fait 5 échantillons récoltés par placette. Au total, 630 carrés de rendement ont été récoltés à l'échelle de cette aire de pâturage. La détermination de la matière sèche a été faite à l'aide de 5 échantillons par transect (ce qui fait 20 échantillons) choisis au hasard. Le séchage a été fait à l'étuve réglée à $105{ }^{\circ} \mathrm{C}$ pendant 48 heures.

\section{Relevé et estimation de la phytomasse ligneuse}

Au niveau de chaque placette, il a été procédé au dénombrement exhaustif des individus de chaque espèce. Après avoir établi la liste des espèces ligneuses, des mesures sur les paramètres dendrométriques ont été réalisées. Ainsi sur chaque individu ligneux, les mesures ont porté sur les diamètres du tronc à $0,3 \mathrm{~m}$ et à $1,3 \mathrm{~m}$; le diamètre du houppier dans deux directions (Est-Ouest et Nord-Sud); la hauteur et le nombre de rejets (vivants et morts). Ceci a permis d'estimer la densité des ligneux, la surface terrière, le couvert aérien, la structure du peuplement ligneux et la régénération des espèces.

\section{Traitement et analyse des données}

Toutes les données ont été saisies avec le tableur Excel. Différents paramètres ont été 
calculés, des tableaux et figures ont été réalisés:

- la diversité floristique (familles, genres, espèces) a été appréciée à partir de la liste floristique générale des espèces inventoriées;

- la fréquence spécifique (Fsi) d'une espèce a été obtenue en faisant la somme des présences de cette espèce dans chaque relevé;

- la contribution spécifique d'une espèce (Csi) correspond à la proportion de cette espèce, exprimée en pourcentage. Elle est donnée par la formule suivante : Csi $(\%)=$ (Fsi $/ \Sigma$ Fsi $) * 100$

- la valeur pastorale $(\mathrm{Vp})$ a été calculée en multipliant les contributions des espèces (Csi) par les indices de qualité correspondants (Isi); les valeurs pastorales relatives (Vpr) des espèces ainsi obtenues sont additionnées puis divisées par le nombre de classes significatives d'indices (Isi $\neq 0$ ) et exprimées en pourcentage (Akpo et Grouzis, 2000): Vp $=1 / 3 \Sigma$ (Csi * Is);

- la valeur pastorale nette est appliquée à la phytomasse produite pour qualifier le fourrage produit (Akpo et Grouzis, 2000) : $\mathrm{Q}(\mathrm{kg} \mathrm{MS} / \mathrm{ha})=\mathrm{P} * \mathrm{Vp}$ avec Q: la quantité de phytomasse totale qualifiée, $\mathrm{P}$ est la production résiduelle en kilogrammes de matière sèche par hectare ;

- la capacité de charge est un rapport qui permet de déterminer jusqu'à quel niveau un pâturage peut être exploité sans risque de dégradation ou de sous-alimentation du bétail (Breman et Ridder, 1991). Elle peut être exprimée soit en nombre d'unité de bétail tropicale (UBT) par hectare pâturé, soit en nombre d'hectares nécessaires pour nourrir une UBT pendant une période donnée (Beagges, 2003). Selon Boudet (1984), le poids des bovins tropicaux oscille entre 200 à $400 \mathrm{~kg}$ ce qui permet de définir l'unité bovin tropical (UBT) comme correspondant à un bovin de 250 $\mathrm{kg}$ à l'entretien. Aussi, selon ce même auteur, on estime à $1 / 3$ de la biomasse primaire, le disponible fourrager réellement consommable par les bovins et à $6,25 \mathrm{~kg}$ la consommation journalière en matière sèche de l'UBT en pâturage sans contrainte. Dans l'estimation de la capacité de charge de cette étude, l'hypothèse que l'UBT (Unité de Bétail Tropical de $250 \mathrm{~kg}$ de poids vif) doit consommer une quantité de $6,25 \mathrm{~kg}$ de matière sèche par jour est adoptée. Ainsi la charge animale pour une période donnée est calculée comme suit : $\mathrm{UBT} / \mathrm{ha}=$ (Production de phytomasse totale qualifiée (kg MS/ha) x k) / 6,25 (kg MS/UBT) avec $6,25=$ consommation journalière d'une UBT; $\mathrm{k}=$ coefficient d'utilisation $(\mathrm{k}=0,33)$; MS : matière sèche ;

- la fréquence de présence renseigne sur la distribution d'une espèce dans les stations. Elle est exprimée par la formule: $\mathrm{F}=$ $(\mathrm{Ni} / \mathrm{Nr})^{*} 100$ avec $\mathrm{F}=$ fréquence de présence $(\%) ; \mathrm{Ni}=$ effectif total de l'espèce i, $\mathrm{Nr}=$ effectif total du peuplement.

- la densité d'une espèce ligneuse correspond au nombre de pieds appartenant à cette espèce par unité de surface $\left(n \cdot h^{-1}\right)$. Elle est exprimée par la formule : $\mathrm{D}=\mathrm{Ni} / \mathrm{S}$ avec $\mathrm{D}=$ Densité; $\mathrm{Ni}=$ effectif total de l'espèce $i$ dans l'échantillon considéré et $\mathrm{S}=$ surface de l'échantillon en ha ;

- la surface terrière désigne la proportion de la surface échantillon occupée ou l'aire d'encrage (évaluée à la base) d'un individu. Elle a été calculée selon l'expression: $\mathrm{St}=\Sigma \pi\left(\mathrm{d}_{0}, 3 / 2\right)^{2} / \mathrm{S}$ avec $\mathrm{St}=$ surface terrière $; \mathrm{d}_{0,3}=$ diamètre en $\mathrm{m} \mathrm{du}$ tronc à $0,3 \mathrm{~m}$ du sol, $\mathrm{S}=$ surface de l'échantillon considéré en ha ;

- le couvert aérien correspond à la proportion verticale de la surface d'un échantillon occupée au sol par la couronne d'un végétal : $\mathrm{Ca}=\Sigma \pi(\mathrm{dmh} / 2)^{2} / \mathrm{S}$ avec $\mathrm{Ca}$ $=$ couvert aérien $; \mathrm{dmh}=$ diamètre moyen du houppier en $\mathrm{m}$, qui est égal à la moitié de la somme des diamètres Nord-Sud et Est-Ouest ; $\mathrm{S}=$ surface de l'échantillon considéré en ha ;

- la structure du peuplement ligneux a été établie avec des classes de hauteur et de diamètre à $1,3 \mathrm{~m}$. 


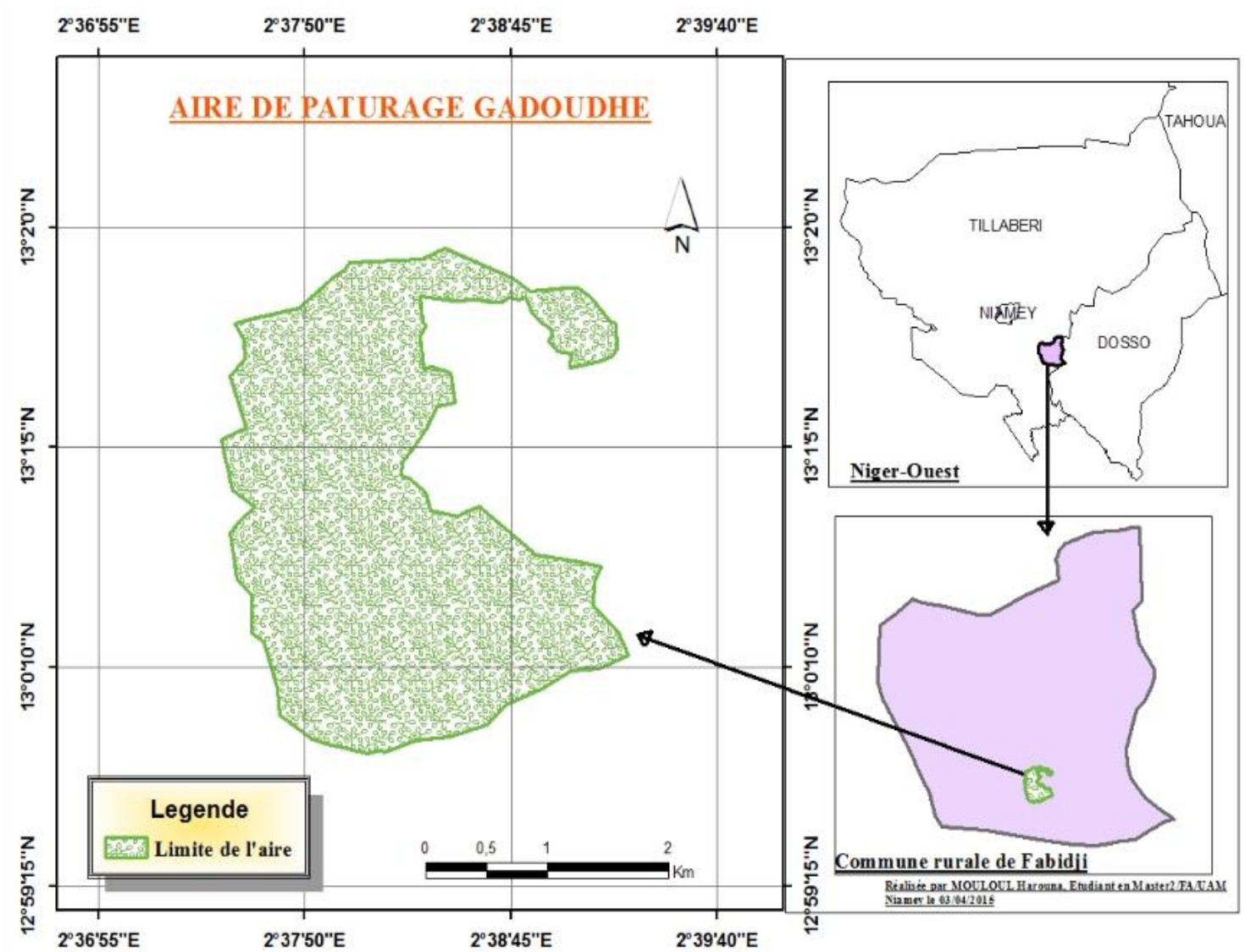

Figure 1: Localisation du parcours Gadoudhé dans la commune de Fabidji (Niger).

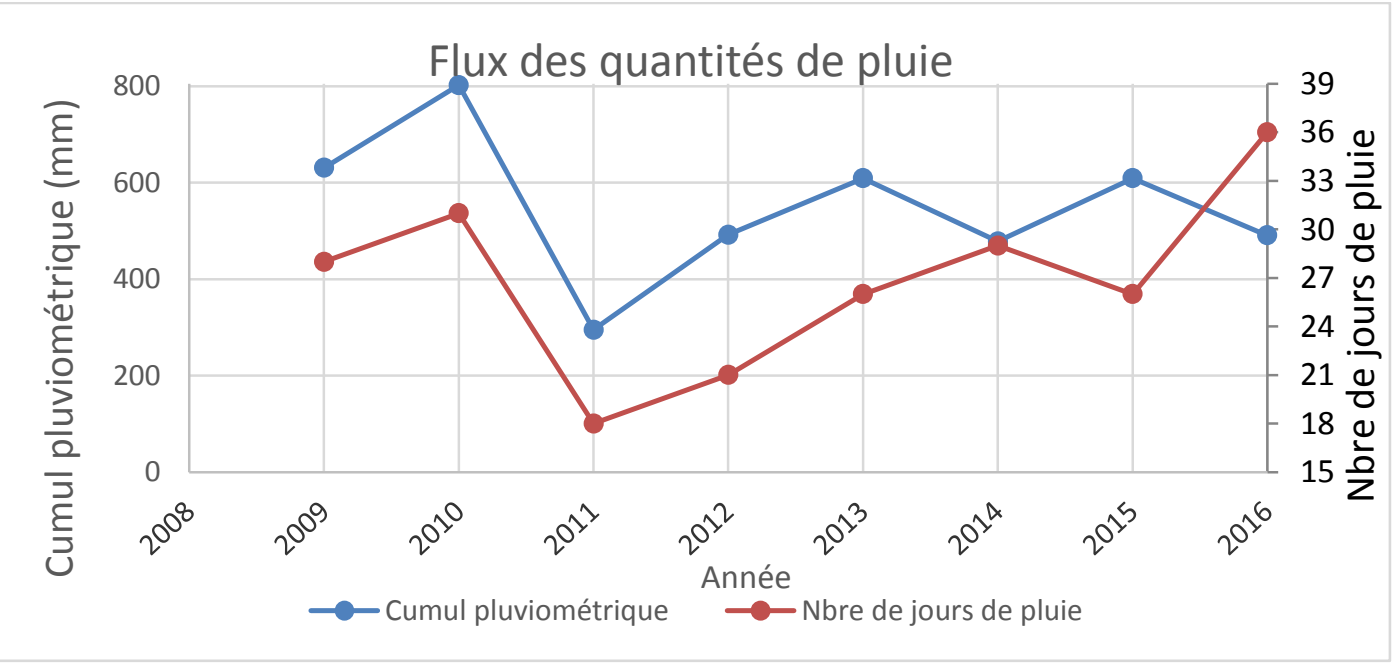

Figure 2: Variation des quantités de pluies enregistrées dans la commune rurale de Fabidji durant les 8 dernières années (2009-2016). 


\section{RESULTATS}

\section{Composition floristique du parcours}

La composition floristique du parcours est résumée dans le tableau 1 . Il ressort de ce tableau que le parcours est riche de 27 espèces herbacées, réparties en 25 genres et 16 familles. Les familles les plus représentées sont les Fabacées (3 espéces), les Poacées (3 espéces), les Amaranthacées (3 espéces), les Convolvulacées (3 espéces), les Cucurbitacées ( 2 espéces), les Molluginacées ( 2 espéces) et les Cyperacées (2 espéces). Les autres familles sont représentées par une seule espèce.

La part des ligneux est consignée dans le tableau 2. Il a été recensé 10 espèces reparties en 7 genres et 6 familles. La famille des Combrétacées est la plus représentée (3 espèces), suivie des Mimosacées (2 espéces) et des Anacardiacées ( 2 espéces). Les autres familles sont représentées par une seule espèce.

\section{Production fourragère, capacité de charge et valeur pastorale (ou indice global de qualité)}

Le tableau 3 indique les valeurs de la production fourragère, de la capacité de charge et la proportion de la valeur pastorale. Il ressort de ce tableau que la quantité de fourrage « qualifié » équivaut à 283,61 kg de MS/ha. Ce qui correspond à une capacité de charge estimée à $0,041 \mathrm{UBT} / \mathrm{ha} / \mathrm{an}$. La valeur pastorale du parcours est de $60,20 \%$. Elle montre une dominance des espéces à indice spécifique de qualité importante.

\section{Densité et recouvrement du peuplement ligneux}

Le parcours présente une densité très faible. Elle est de 13,75 individus/ha. Le recouvrement est aussi faible avec une surface terrière de $0,01 \mathrm{~m}^{2} /$ ha et un couvert aérien de $10,15 \mathrm{~m}^{2} / \mathrm{ha}$.

\section{Structure du peuplement ligneux}

Le peuplement ligneux est majoritairement constitué des individus dont la hauteur appartient aux classes] 5- 7m] (37\%),] 7-9m] (29\%) et] 3-5m] (27\%). Les individus de petite taille (hauteur $\leq 3 \mathrm{~m}$ ) et les individus de grande taille (hauteur $>9 \mathrm{~m}$ ) sont rares. Chez les deux espèces dominantes (Combretum nigricans et Lannea microcarpa), les sujets dont la hauteur est comprise entre 5 et $7 \mathrm{~m}$ sont majoritaires $(39,73 \%)$. Il existe au sein de ces deux espèces dominantes, quelques arbres dont la hauteur est $>9 \mathrm{~m}$ (Figure 3 ).

Les arbres dont les diamètres appartiennent aux classes] $10-15 \mathrm{~cm}$ ] $(42 \%)$,] $5-10 \mathrm{~cm}](37 \%)$; sont majoritaires. Les gros arbres (avec des diamètres >15 cm) sont minoritaires. Combretum nigricans est surtout représenté aux classes de diamètre] $5-10 \mathrm{~cm}$ ] et] $10-15 \mathrm{~cm}$ ]. Lannea microcarpa est représenté dans presque toutes les classes avec une dominance des classes] $15-20 \mathrm{~cm}$ ] et] 20 - $25 \mathrm{~cm}$ ]. Lannea microcarpa présente quelques gros individus avec des diamètres > $25 \mathrm{~cm}$ (Figure 4).

\section{La régénération des espèces}

La régénération des jeunes plants est consignée dans le tableau 4. Il ressort du tableau qu'elle est très active. En effet, il a été recensé 32159 jeunes plants vivants et 4886 morts répartis dans 9 familles et 11 genres. La famille des combrétacées est la plus représentée avec 4 espèces. La densité de la régénération est de 1020,91 rejets à l'hectare. 
Tableau 1: Caractéristiques floristiques des herbacés du parcours Gadoudhé.

\begin{tabular}{|c|c|c|c|c|}
\hline Famille & Espèce & Csi \% & Is & Vpr\% \\
\hline \multirow[t]{3}{*}{ Fabaceae } & Zornia glochidiata & 14,59 & 2 & 29,18 \\
\hline & Aeschynomene indica & 0,20 & 0 & 0,00 \\
\hline & Tephrosia purpurea & 0,01 & 3 & 0,03 \\
\hline Malvaceae & Sida cordifolia & 1,61 & 0 & 0,00 \\
\hline Commelinaceae & Commelina benghalensis & 30,83 & 2 & 61,66 \\
\hline Rubiaceae & Mitracarpus scaber & 0,84 & 0 & 0,00 \\
\hline \multirow[t]{3}{*}{ Amaranthaceae } & Pupallialappacea & 7,66 & 1 & 7,66 \\
\hline & Pandiakaheudelotii & 0,24 & 1 & 0,24 \\
\hline & Amaranthusspinosus & 0,04 & 0 & 0,00 \\
\hline \multirow[t]{3}{*}{ Poaceae } & Aristida mutabilis & 38,29 & 2 & 76,58 \\
\hline & Cenchrus biflorus & 0,01 & 3 & 0,03 \\
\hline & Pennisetum pedicellatum & 0,05 & 2 & 0,10 \\
\hline Acanthaceae & Monechmaciliatum & 4,58 & 1 & 4,58 \\
\hline \multirow[t]{2}{*}{ Cyperaceae } & Fimbristylisspp & 0,04 & 2 & 0,08 \\
\hline & Cyperus amabilis & 0,13 & 2 & 0,25 \\
\hline \multirow[t]{2}{*}{ Molluginaceae } & Mollugocerviana & 0,28 & 0 & 0,00 \\
\hline & Mollugonudicaulis & 0,06 & 0 & 0,00 \\
\hline \multirow[t]{3}{*}{ Convolvulaceae } & Ipomoeaasarifolia & 0,03 & 0 & 0,00 \\
\hline & Merremia pinnata & 0,01 & 2 & 0,02 \\
\hline & Jacquemontiatamnifolia & 0,04 & 1 & 0,04 \\
\hline Caryophyllaceae & Polycarpaeaspp & 0,25 & 0 & 0,00 \\
\hline Caesalpiniaceae & Cassia mimosoides & 0,01 & 1 & 0,01 \\
\hline \multirow[t]{2}{*}{ Cucurbitaceae } & Cucumismelo & 0,07 & 1 & 0,07 \\
\hline & Cucumisprophetarum & 0,01 & 1 & 0,01 \\
\hline Curcubitaceae & Citrulluslanatus & 0,02 & 2 & 0,04 \\
\hline Aizoaceae & Limeuтpterocarpum & 0,03 & 1 & 0,03 \\
\hline Euphorbiaceae & Phyllanthus pentandrus & 0,07 & 0 & 0,00 \\
\hline
\end{tabular}

Tableau 2 : Liste des espèces ligneuses inventoriées dans le parcours Gadoudhé.

\begin{tabular}{llcc}
\hline Famille & \multicolumn{1}{c}{ Espèce } & Effectif & Fréquence(\%) \\
\hline Combretaceae & Combretum nigricans & 344 & 79,45 \\
& Combretum glutinosum & 2 & 0,46 \\
Rubiaceae & Combretum micranthum & 8 & 1,85 \\
Capparidaceae & Gardiena erubescens & 1 & 0,23 \\
Mimosaceae & Maerua crassifolia & 1 & 0,23 \\
& Albizzia chevalieri & 1 & 0,23 \\
\hline
\end{tabular}




\begin{tabular}{llcc}
\hline & Acacia macrostachya & 1 & 0,23 \\
Caesalpiniaceae & Cassia sieberana & 1 & 0,23 \\
Anacardiaceae & Lannea microcarpa & 73 & 16,86 \\
& Sclerocarya birrea & 1 & 0,23 \\
Total & & 433 & 100 \\
\hline
\end{tabular}

Tableau 3: Caractéristiques des herbacés du parcours Gadoudhé.

\begin{tabular}{llllclll}
\hline $\begin{array}{l}\text { Parcours } \\
\text { Gadoudhé }\end{array}$ & $\begin{array}{l}\text { Valeur } \\
\text { pastorale } \\
(\boldsymbol{\%})\end{array}$ & $\begin{array}{l}\text { Quantité de } \\
\text { phytomasse } \\
\text { brute }(\text { Kg de } \\
\text { MS/ha) }\end{array}$ & $\begin{array}{l}\text { Quantité } \\
\text { phytomasse } \\
\text { «qualifiée» } \\
\text { de MS/ha) }\end{array}$ & $\begin{array}{l}\text { Nombre } \\
\text { de jours } \\
\text { de pâture }\end{array}$ & $\begin{array}{l}\text { Capacité } \\
\text { de charge } \\
\text { (UBT } \\
\text { /ha/an) }\end{array}$ & $\begin{array}{l}\text { Capacité de } \\
\text { charge } \\
\text { (ha/UBT/an) }\end{array}$ \\
\hline $\begin{array}{l}\text { Végétation } \\
\text { herbacée }\end{array}$ & 60,20 & 471,1 & 283,61 & 15 & 0,041 & 24,39 \\
\hline
\end{tabular}

Tableau 4 : Régénération des espèces ligneuses du parcours Gadoudhé.

\begin{tabular}{|c|c|c|c|c|}
\hline Famille & Espèce & $\begin{array}{l}\text { Vivante } \\
\text { (nombre d'individus) }\end{array}$ & $\begin{array}{l}\text { Morte } \\
\text { (nombre d'individus) }\end{array}$ & $\begin{array}{l}\text { Densité } \\
\text { (rejets/ha) }\end{array}$ \\
\hline \multirow[t]{4}{*}{ Combretaceae } & $\begin{array}{l}\text { Combretum } \\
\text { micranthum }\end{array}$ & 10903 & 1879 & 346,13 \\
\hline & $\begin{array}{l}\text { Guiera } \\
\text { senegalensis }\end{array}$ & 8872 & 1564 & 281,65 \\
\hline & $\begin{array}{l}\text { Combretum } \\
\text { nigricans }\end{array}$ & 861 & 257 & 27,33 \\
\hline & $\begin{array}{l}\text { Combretum } \\
\text { gluttinosum }\end{array}$ & 116 & 1 & 3,68 \\
\hline Rubiaceae & $\begin{array}{l}\text { Gardenia } \\
\text { erubescens }\end{array}$ & 8397 & 643 & 266,57 \\
\hline \multirow[t]{2}{*}{ Capparidaceae } & $\begin{array}{l}\text { Boscia } \\
\text { senegalensis }\end{array}$ & 1785 & 369 & 56,66 \\
\hline & $\begin{array}{l}\text { Maerua } \\
\text { crassifolia }\end{array}$ & 828 & 128 & 26,29 \\
\hline Polygalaceae & $\begin{array}{l}\text { Securidaca } \\
\text { longepedunculata }\end{array}$ & 18 & 1 & 0.57 \\
\hline Mimosaceae & $\begin{array}{l}\text { Acacia } \\
\text { macrostachya }\end{array}$ & 235 & 36 & 7,46 \\
\hline Olacaceae & $\begin{array}{l}\text { Ximenia } \\
\text { americana }\end{array}$ & 135 & 8 & 4,29 \\
\hline
\end{tabular}




\begin{tabular}{llllc}
\hline Caesalpiniaceae & $\begin{array}{l}\text { Cassia } \\
\text { sieberana }\end{array}$ & 1 & 0 & 0,03 \\
& $\begin{array}{l}\text { Piliostigmareticul } \\
\text { atum }\end{array}$ & 2 & 0 & 0.06 \\
Tiliaceae & Grewiabicolor & 5 & 0 & 0.16 \\
Anacardiaceae & $\begin{array}{l}\text { Lannea } \\
\text { microcarpa }\end{array}$ & 1 & 0 & 0.03 \\
Total & 32159 & 4886 & 1020,91 \\
\hline
\end{tabular}
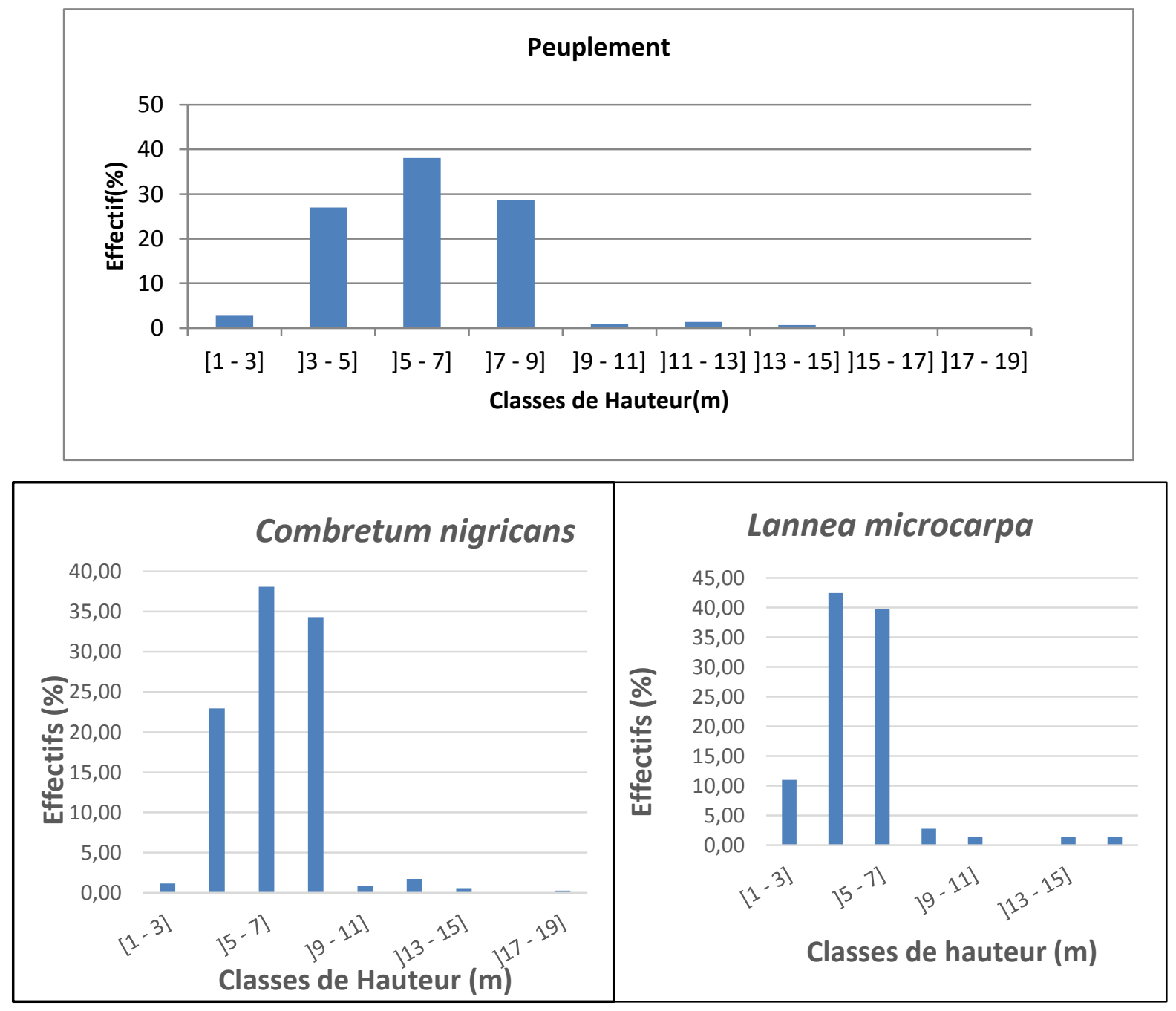

Figure 3 : Distribution des effectifs du peuplement et des deux espèces dominantes en fonction des classes d'hauteur. 


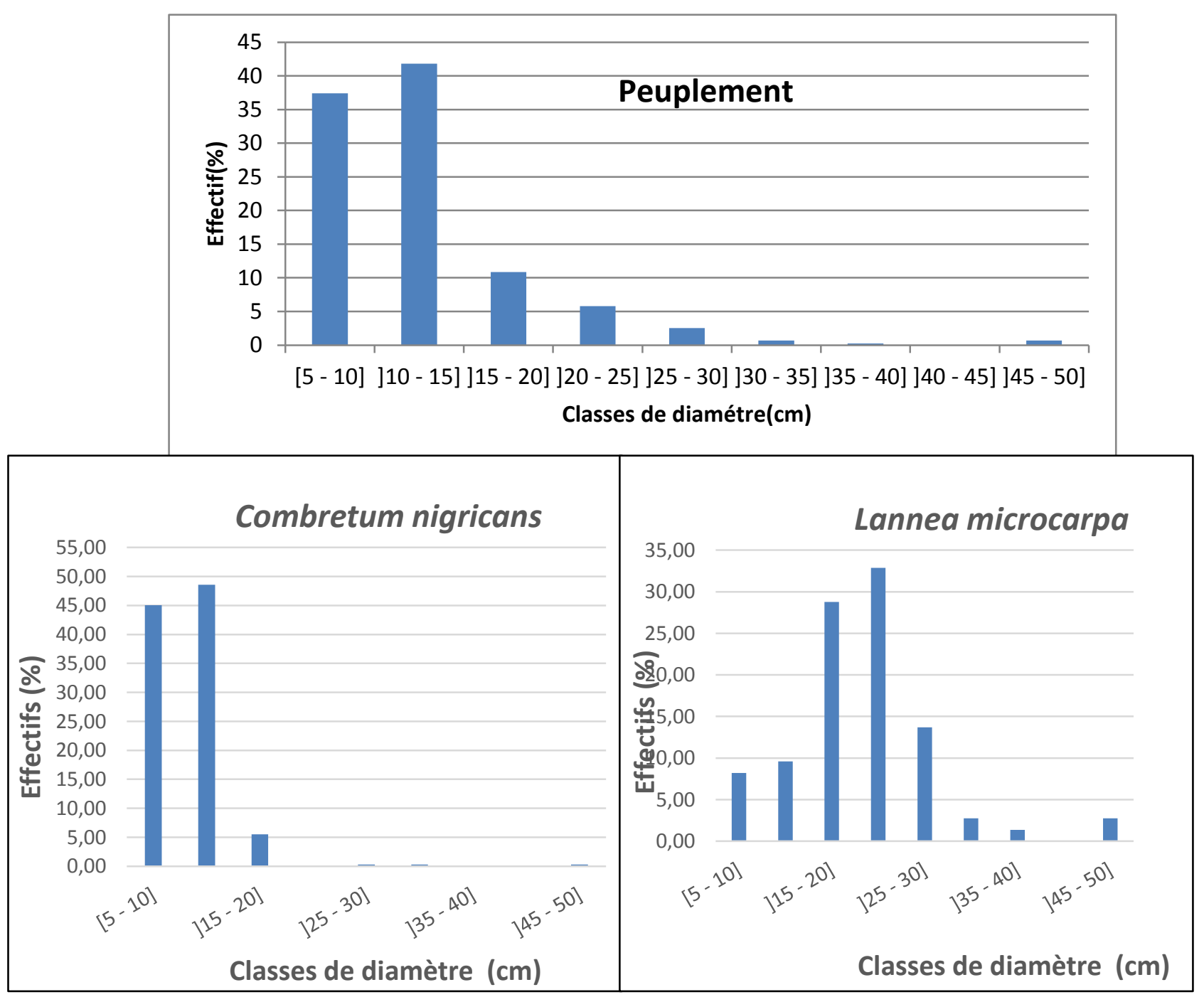

Figure 4: Distribution des effectifs du peuplement et de deux des espèces dominantes en fonction du diamètre.

\section{DISCUSSION}

La composition floristique du parcours Gadoudhé est caractérisée par une dominance de Poacées (Aristida mutabilis, Cenchrus biflorus, Pennisetum pedicellatum). Les travaux antérieurs sur la composition floristique de végétation herbacée des savanes soudano-sahéliennes ont révélé qu'elle est très largement dominée par les graminées annuelles (Akpo et al., 2003). Cette dominance des graminées annuelles est une réponse aux effets conjugués de l'aridité et de la pression de pâture (Akpo et Grouzis, 200). En effet, les graminées sont des espèces qui résistent aux différents chocs en développant une stratégie leur permettant de se maintenir dans un environnement perturbé.

L'estimation de la production de phytomasse des pâturages sahéliens dépend fortement du cortège floristique des pâturages, qui co-détermine la quantité et la qualité du fourrage disponible. Ainsi, la phytomasse de 
471,1 kg MS/ha enregistrée dans le parcours Gadoudhé, avec une valeur pastorale nette de $60,20 \%$ n'équivaut qu'à $283,61 \mathrm{~kg}$ de $\mathrm{MS} / \mathrm{ha}$ de fourrage «qualifié ». Cette pondération « qualité » du fourrage fiabilise l'estimation de la capacité de charge en bétail (Akpo et Grouzis, 2000). Par ailleurs, la capacité de charge constitue un bon indicateur de gestion durable du bilan fourrager. En effet, le rapport de la charge réelle à la capacité de charge constitue un bon indice d'intensité d'exploitation du pâturage; il y a surexploitation lorsqu'il est supérieur à 1 (Allen et al., 2011). Dans le parcours Gadoudhé où la production de fourrage «qualifiée » est de 283,61 kg de MS/ha, il faut donc 24,39 ha pour entretenir une UBT/an ou 0, $041 \mathrm{UBT} / \mathrm{ha} / \mathrm{an}$. Cette aire de pâturage de la commune de Fabidji (Niger) est dès lors surexploitée en tenant compte de l'effectif du cheptel.

La valeur de capacité de charge de 0,041 UBT/ha/an obtenue dans cette étude, est faible par rapport à celle de $0,41 \mathrm{UBT} / \mathrm{ha} / \mathrm{an}$ rapportée par Ngom et al. (2012) dans la zone tampon de la réserve de biosphère du Ferlo au Sénégal. La différence entre ce résultat et celui de cet auteur, s'explique probablement par le fait que sa zone d'étude est une réserve, alors que la zone de cette étude est peuplée avec une densité humaine relativement élevée de 43,86 habitants $/ \mathrm{km}^{2}$ (INS, 2014) et dont $89,6 \%$ des éleveurs sédentaires sont en permanence dans le parcours (Djibo et al., 2016).

Dans le parcours Gadoudhé, la végétation ligneuse renferme 10 espèces avec une forte dominance de la famille des Combrétacées (Combretum nigricans, Combretum glutinosum, Combretum micranthum). Une telle dominance de cette famille a été rapportée par Ngom (2008) dans la zone du Ferlo (Nord- Sénégal).

La surface terrière a été évaluée à 0,01 $\mathrm{m}^{2} /$ ha pour une densité de 13,75 individus à l'hectare. Cette valeur est très faible par rapport à celle trouvée par Douma et al. (2007) qui est de $1,6 \mathrm{~m}^{2} /$ ha pour une densité de 178,5 individus à l'hectare à la station sahélienne expérimentale de Toukounous au Niger et par rapport à celle enregistrée par Ndiaye et al. (2010) qui est de $4,04 \mathrm{~m}^{2} / \mathrm{ha}$ pour une densité de 288 individus à l'hectare dans les terroirs pastoraux de Ranérou au Sénégal. La différence entre ce résultat et ceux de ces auteurs, peut s'expliquer par le fait que leurs zones d'étude sont nettement moins pâturées que le parcours Gadoudhé. En effet, ce dernier représente la principale zone de pâture du terroir avec une forte proportion du bétail (environ 2500 têtes par espèce de ruminants) fréquentant régulièrement le parcours (Djibo et al., 2016).

La distribution des individus par classe de hauteur ou de diamètre a montré que les individus de taille moyenne et de diamètre moyen constituent l'essentiel du peuplement et confirme ainsi l'exploitation de ces ressources ligneuses. Une telle distribution met aussi en évidence un réel potentiel de régénération (Ndiaye et al., 2010). En effet, la régénération des jeunes plants est active avec une densité de 1020,91 rejets à l'hectare. Une régénération qui est malheureusement beaucoup plus importante chez les espèces peu ou pas appétées (Combretum micranthum, Guiera senegalensis et Gardenia erubescens).Ces espèces sont en fait bien adaptées aux conditions du milieu.

\section{Conclusion}

Les résultats de la présente étude montrent que 37 espèces ont été répertoriées au niveau du parcours dont 27 sont des herbacés. Les familles les plus représentées sont les Poacées, les Fabacées, les Amaranthacées et les Convolvulacées. La phytomasse brute est de 471,1 kg MS/ha, avec une valeur pastorale nette de $60,20 \%$ qui équivaut à 283,61 $\mathrm{kg}$ de MS/ha de fourrage « qualifié ». Ce qui correspond à une capacité de charge estimée à $0,041 \mathrm{UBT} / \mathrm{ha} / \mathrm{an}$. Quant aux ligneux, ils sont représentés par 10 espèces. La famille des combrétacées est la plus représentée. La structure du peuplement montre des individus de hauteur et de diamètre moyens. La surface terrière a été évaluée à $0,01 \mathrm{~m}^{2} /$ ha pour une densité de 
13,75 individus à l'hectare. La régénération des jeunes plants est active avec une densité de 1020,91 rejets à l'hectare. Au total, les caractéristiques de la végétation (herbacée et ligneuse) montrent que les ressources fourragères disponibles ne peuvent plus assurer une alimentation adéquate du bétail. Il est par conséquent nécessaire de procéder à un aménagement du parcours en collaboration avec les éleveurs afin de permettre une exploitation rationnelle et durable des ressources végétales pour la satisfaction des besoins alimentaires du bétail.

\section{CONFLIT D'INTERETS}

Les auteurs de ce manuscrit déclarent qu'il n'y a aucun conflit d'intérêt entre eux.

\section{CONTRIBUTIONS DES AUTEURS}

Dans la présente étude, Monsieur Ibrahim Djibo a assuré l'élaboration du protocole de recherche, la collecte, le traitement des données et la rédaction du manuscrit sous l'encadrement et la supervision de Monsieur Mani Mamman, Monsieur Chaibou Issa et Monsieur Oumar Sarr. Madame Amy Bakhoum, Monsieur Hamani Marichatou, Monsieur Léonard .E. Akpo et Monsieur Moussa Assane ont contribué à la relecture des différentes versions pour l'amélioration de la qualité scientifique du manuscrit.

\section{REMERCIEMENTS}

Les auteurs remercient le Programme de Productivité Agricole en Afrique de l'Ouest (PPAAO) pour le financement de cette étude.

\section{REFERENCES}

Akpo LE, Grouzis M. 2000. Valeur pastorale des herbages en région soudanienne, cas des parcours sahéliens du Nord-Sénégal. Tropicultura, 18 : 1-8.

Akpo LE, Banoin M, Grouzis M. 2003. Effet de l'arbre sur la production et la qualité fourragères de la végétation herbacée : bilan pastoral en milieu sahélien. Rev.
Elev. Méd. Vét. Pays Tropicaux, 154(10): 619-628.

Allen VG, Batello C, Berretta EJ, Hodgson J, Kothmann M, Li X, Mclvor J, Milne J, Morris C, Peeters A, Sanderson M. 2011. An international terminology for grazing lands and grazing animals. Grass and Forage Science, 66: 2-28. DOI: https://doi.org/10.1111/j.13652494.2010.00780.x .

Beagges S. 2003. Caractéristiques sociologiques et socio pastorales des villages de la zone du projet moyen Bani. Bamako, Mali, 32 p.

Boudet G.1984. L'exploitation des parcours et la conduite des troupeaux dans les systèmes d'élevage. Les Cahiers de la Recherche-Développement, 3-4 : 97-101.

Breman H, De Ridder N. 1991. Manuel sur les Pâturages des Pays Sahéliens, Karthala, ACCT, CABO-DLO et CTA, 485p.

Chaibou M, Yaou OM, Gouro A, Laouali A. 2012. Diversité, disponibilité et circuits d'approvisionnement des aliments du bétail dans la communauté urbaine de Maradi. Journal des Sciences de l'Environnement, 1(1) : 27-34.

Daget P, Godron M. 1995. Pastoralisme. Troupeaux, Espaces et Sociétés. HatierAupelf.Uref : Paris, France; p. 206-218.

Daget P, Poissonet J. 1971. Une méthode d'analyse phytologique des prairies. Critères d'application. Ann Agron, 22 : 5-41.

Devineau JL, Leordier C, Vuattoux R. 1984. Evolution de la diversité spécifique du peuplement ligneux dans une succession preforestière de colonisation d'une savane protégée des feux (Lamto, Côte d'Ivoire). Conservatoire et Jardin Botanique de la Ville de Genève, 39 : 103-133.

Djibo I, Mamman M, Bakhoum A, Sarr O, Marichatou H, Akpo LE, Assane M. 2016. Évaluation de l'importance du parcours Gadoudhé, dans l'alimentation du bétail de la commune rurale de 
Fabidji au Niger. Journal of Applied Biosciences, 106: 10266 -10273. DOI : http://dx.doi.org/10.4314/jab.v106i1.6.

Douma S, Diatta S, Kabore-Zoungrana CY, Banoin M, Akpo LE. 2007. Caractérisation des terres de parcours sahéliennes: typologie du peuplement ligneux de la Station sahélienne Expérimentale de Toukounous au Niger. Journal des Sciences, 7: 1-16.

Garba I, Touré I, Ickowicz A, Ancey V, Akpo EL, Ba A, Coenu C, Diop AT, Gaye IJ, Leclerc G, Ndiaye P, Niang I, Saley M, Soumane MA, Toutain B, Wane A. 2009. Système d'Information sur le Pastoralisme au Sahel. Programme LEAD, Rapport final, deuxième phase, Octobre 2009.- 104p.

FAO. 2003. World Agriculture: towards.2015/2030. An FAO pespectives. FA, Ed. J Bruinsma Earths can Publications, Ltd, London.-407p.

FAO. 2006. World Agriculture: towards 2030/ 2 50. Interim report Porspects for food, nutrition, agriculture and major commodity groups. Global perspectives Studies. Rome.- 78p.

Maidadji B. 2003. L'élevage au Niger: systèmes en place, politiques commerciales, atouts et limites. In: Quelles politiques pour améliorer la compétitivité des petits éleveurs dans le corridor central de l'Afrique de l'ouest: implications pour le commerce et l'intégration régionale, Ehui $\mathrm{S}$, Barry MB, Williams TO, Koffi KM, Zeleka P (eds). Proceedings of a workshop held in Abidjan, Côte d'Ivoire, 17-18 September 2001. ILRI (Institut international de recherche sur l'élevage), Nairobi, Kenya.-88 p.

N'Diaye M, Dione ME, Akpo LE. 2010. Caractéristiques des ligneux dans les terroirs pastoraux du Ranérou (Région de Matam, Nord-Sénégal). Journal des Sciences, 10(3) : 12-27.

Ngom D, Bakhoum A, Diatta S, Akpo LE. 2012. Qualité pastorale des ressources herbagères de la réserve de biosphère du Ferlo (Nord-Sénégal). Int J. Biol. Chem. Sci., $\quad$ 6(1): 186-201. DOI: http://dx.doi.org/10.4314/ijbcs.v6i1.17.

Ngom D. 2008. Définition d'indicateurs de gestion durable des ressources sylvopastorales au Ferlo (NordSénégal), Doct. 3C, Biologie végétale, UCAD, Dakar, 148p.

Pagot J. 1985. L'Elevage en Pays Tropicaux. Maisonneuve et Larose, ACCT : Paris, France ; $526 \mathrm{p}$.

Rhissa Z. 2010. Revue du secteur de l'élevage au Niger, Rapport provisoire Février 2010.-Niamey : FAO/SFW.-115p.

Uwizeye A. 2008. Impact du changement climatique sur les productions animales. Thèse: Med. Vet, UCAD, Dakar, p.46. 